\title{
Zum Nutzen der Frame-Semantik für die Analyse der Bedeutungskonstitution in der Interaktion
}

\section{Einleitung}

Dieser Beitrag nimmt die Eignung eines kognitiven Ansatzes, der Frame-Semantik, für interaktionslinguistische Bedeutungsanalysen in den Blick. Frames gelten als mit Lexemen assoziierte konzeptuelle Strukturen, die Interpretationen von Åußerungen zugrundeliegen (vgl. Fillmore 1985). Sie werden von Linguisten für gewöhnlich introspektiv ermittelt und anhand von Korpusdaten allenfalls auf Plausibilität geprüft (vgl. z. B. Ruppenhofer et al. 2010). Es soll anhand zweier Phänomenbereiche diskutiert werden, inwiefern sich die Relevanz der vom Analytiker angenommenen Frames auch für die Interaktionsteilnehmer zeigen lässt. Als erstes Beispiel wird der Standardfall der von Verben evozierten Frames betrachtet. Es wird analysiert, inwiefern sich anhand des sequenziellen Kontexts eines Einzelfalls oder anhand rekurrenter Muster zeigen lässt, dass einzelne (nicht immer overt als Verbergänzungen realisierte) Frame-Elemente mit konstitutiv für die Bedeutung sind. Als zweites Beispiel dient die Verwendung von Diskurspartikeln. Da diesen trotz ihrer Kontextabhängigkeit in der Regel eine Grundbedeutung zugeschrieben wird, insbesondere wenn es sich um aus autosemantischem Material stammende Vertreter (z. B. komm, schau) handelt, stellt sich die Frage, ob eine framesemantische Beschreibung dieser Grundbedeutung (z. B. ein Bezug auf den für kommen relevanten Motion-Frame bei komm) gegenüber anderen (kognitiven und nicht-kognitiven) semantischen Ansätzen einen Mehrwert bietet und sich empirisch rechtfertigen lässt. Dabei zeigt sich auch, wo (kognitiv-)linguistische Semantiktheorien an ihre methodischen Grenzen stoßen: bedeutungskonstitutiv relevante Inferenzen können nicht immer durch (mentale) Zuschreibungen des Analytikers ermittelt werden, sondern müssen oft dem sequenziellen Kontext oder ethnographischem Wissen entnommen werden. Dies zeigt, dass nicht nur die Frame-Semantik interaktionslinguistische Ansätze bereichern kann, indem sie z. T. nur introspektiv zugängliche Bedeutungsanteile kognitiv angemessen beschreibbar macht, sondern umgekehrt auch interaktionale Analysen kognitiven Theorien neue Impulse geben können, da sie darauf 
verweisen, dass Bedeutungskonstitution immer mehr als die propositionale Ebene und rein enzyklopädische Wissensbestände umfasst.

\section{Theoretische Grundlagen}

\subsection{Interaktionslinguistische Ansätze und Kognition}

Pragmalinguistische Ansätze, die sich mit gesprochener Sprache beschäftigen, sehen sich selbst häufig als nicht kompatibel mit kognitiven Ansätzen, seien sie psychologisch oder linguistisch orientiert. Insbesondere viele Gesprächsanalytiker betonen die ,antimentalistische' Ausrichtung ihrer Methode (vgl. im Überblick Potter/Edwards 2013): Bei Sequenzanalysen von Transkripten wird nur einbezogen, was ,beobachtbar' ist, d.h. die für alle Teilnehmer der untersuchten Interaktion zugänglichen Redebeiträge, mit denen sie nur teilweise ihre Absichten offen ,anzeigen' (zum display-Begriff vgl. z. B. Sacks/Schegloff/Jefferson 1974: 728). Auch die Interaktionale Linguistik, die neben ihrem Bezug auf die klassische Konversationsanalyse auch von linguistischen Ansätzen (vor allem grammatischen und phonologischen) beeinflusst ist, geht von dem Grundsatz aus, dass den Interagierenden vom Analytiker keine Intentionen und sonstigen mentalen Zustände zugeschrieben werden sollen, die sich nicht aus den Daten ablesen lassen (vgl. z. B. Imo 2014). Dabei bleibt es allerdings immer Interpretationssache, was genau wodurch angezeigt wird und somit ablesbar ist. ${ }^{1}$ Dennoch - oder gerade deshalb - werden auch in vielen interaktionslinguistischen ${ }^{2}$ Analysen Intentionszuschreibungen gemacht und dabei nicht immer (gut) begründet. Dieser Widerspruch zwischen Anspruch und Praxis ist vor allem deshalb problematisch, weil er durch in ihrer Absolutheit unhaltbare Grundannahmen konstruiert wird: Zum einen ist es nicht notwendig, kognitive Ansätze für reine Spekulation zu halten, nur weil man selbst im eigenen Ansatz keine Annahmen zu mentalen Zuständen macht. Es ist unbestritten, dass Menschen solche haben;

1 Es gibt z. B. eindeutige und weniger eindeutige Fälle der „Verstehensdokumentation“ (vgl. Deppermann 2008a): Das Beantworten einer Frage zeigt en passant an, dass sie verstanden worden ist. Ein Rückmeldesignal dagegen zeigt oft zunächst nur an, dass ein Beitrag rezipiert worden ist. Nicht immer ist für den Analytiker erschließbar, was der Produzent eines solchen zu einem gegebenen Zeitpunkt bereits verstanden hat, wenn der Beitrag auf das Rückmeldesignal beschränkt bleibt.

2 Gesprächsanalyse und Interaktionale Linguistik werden hier als ,interaktionslinguistische/ interaktionsanalytische Ansätze‘ zusammengefasst. 
man kann sie wissenschaftlich zu erfassen suchen oder nicht. Wenn man also keine Aussage dazu machen will, welche konkreten mentalen Zustände bei einem Interaktionsteilnehmer vorliegen, negiert man damit nicht auch gleich ihre Existenz. Zum zweiten sind es gerade Intentionszuschreibungen, die menschliche Interaktion und Kommunikation überhaupt erst möglich machen (vgl. z. B. Tomasello 2008), d. h. sowohl die analysierten Interagierenden als auch der Analytiker müssen notwendigerweise Annahmen über mentale Zustände machen, um Interaktionen verstehen zu können. Insofern ist es notwendig, die Grundlagen für die ohnehin gemachten Zuschreibungen genauer zu explizieren und zu reflektieren, anstatt sie als vollkommen unzugänglich darzustellen und zu behandeln. Da Plädoyers für die Offenheit für verschiedene Ansätze und gezielte methodologische Reflexion inzwischen aus der Gesprächsanalyse selbst kommen (vgl. Deppermann 2012; Reineke in diesem Band), soll hier von vornherein von der Möglichkeit einer gegenseitigen Bereicherung ausgegangen werden und, statt eine generelle Kompatibilitätsdiskussion zu führen, ein Bereich herausgegriffen werden, in dem eine Integration kognitiver und nicht kognitiver Ansätze nicht nur wünschenswert, sondern methodologisch notwendig ist.

\subsection{Bedeutungskonstitution in der Interaktion}

Die Semantik ist bisher, anders als z. B. Prosodie und Syntax, sehr selten Gegenstand interaktionslinguistischer Untersuchungen gewesen. Ein Grund dafür ist, dass sie noch weniger ohne introspektive Urteile bzw. durch rein gesprächsanalytische Methoden erfasst werden kann als Syntax und Prosodie, die deutlichere Spuren der Online-Planung hinterlassen. Dadurch lassen sich einige Phänomene auf diesen Ebenen als Teilnehmerkategorien modellieren. Darüber hinaus haben sich trotz des notwendigen Rückgriffs auf traditionelle grammatische und phonologische Konzepte auch genuin interaktionslinguistische Ansätze zum Verständnis dieser Ebenen und vor allem ihrer für die Mündlichkeit spezifischen Phänomene etabliert. Diese betonen, dass ein solches Verständnis allein durch Introspektion nicht erreicht werden kann (z. B. Auers (2000) Ansatz der OnlineSyntax). Bisherige Arbeiten zur Bedeutungskonstitution in der Interaktion beschränken sich weitgehend auf gesprächsanalytische und ethnomethodologische Ansätze, nur vereinzelt werden (kognitiv)linguistische Konzepte diskutiert oder explizit integriert (vgl. Deppermann/Spranz-Fogasy 2002; Deppermann 2007).

Trotz der fehlenden semantiktheoretischen Tradition innerhalb interaktionslinguistischer Ansätze und der dem ,Antimentalismus‘ auf den ersten Blick entgegenstehenden kognitiven Ausrichtung vieler Semantiktheorien gibt es hin- 
sichtlich der Auffassung der Rolle von Kontext bei der Bedeutungskonstitution Gemeinsamkeiten zwischen Gesprächsanalyse und gerade kognitiven - mehr als strukturalistischen - Semantiktheorien (vgl. 2.3). Mit der Verwendung des noch nicht allgemein in der Linguistik terminologisierten Begriffs der Bedeutungskonstitution geht eine Perspektive auf die - notwendigerweise mentale - Verarbeitung von Bedeutung einher, die sie als kontextgesteuerten, interaktiven und/ oder kognitiven Prozess der Herstellung - statt des Abrufens aus einem Lexikon - betrachtet (vgl. Deppermann 2002: 12). Diese umfasst nicht nur propositionsrelevante Anteile:

,Bedeutungskonstitution‘ betrifft Phänomene, die von der Referenz einzelner Ausdrücke, über Sprechakte und die Herstellung von Textkohärenz bis hin zu den Implikationen von Äußerungen für die Sozialbeziehungen der Kommunikationsteilnehmer reichen. (Deppermann 2002: 11)

Während gesprächsanalytische Studien den interaktiven Teil der Herstellung von Bedeutung untersuchen und nichts zum kognitiven Teil des Prozesses sagen, nehmen psychologische Ansätze ebendiesen in den Fokus (vgl. Deppermann 2002). Ein Ansatz, der sich aufgrund der beiden Richtungen gemeinsamen Betonung der Prozesshaftigkeit und der Kontextrelevanz jedoch auch für die interaktionale Analyse der Bedeutungskonstitution anbietet, ist die Frame-Semantik.

\subsection{Frame-Semantik}

Es gibt eine Reihe verschiedener framesemantischer Ansätze bzw. verschiedene Weiterentwicklungen der Idee, die in der Linguistik vor allem auf Charles Fillmore zurückgeht. ${ }^{3}$ Eine Gemeinsamkeit von dessen Theorie mit ethnomethodologischen Ansätzen ist der Einbezug sonst als pragmatisch angesehener Aspekte in die Semantik bzw. das Ablehnen einer klaren Trennung der Semantik von Pragmatik und ,Weltwissen.${ }^{4}$ Verstehensrelevantes Wissen umfasst für ihn mehr

3 Parallel zu Fillmore haben u.a. auch die Kognitionswissenschaftler Minsky und - etwas später - Barsalou Frame-Ansätze entwickelt (vgl. im Überblick Busse 2012).

4 Fillmore selbst verweist am Rande auf diese Gemeinsamkeit - er spricht dabei zwar nicht die Ethnomethodologie, aber ethnographische Perspektiven an: „Frame semantics comes out of empirical semantics rather than formal semantics. It is most akin to ethnographic semantics, the work of the anthropologist who moves into an alien culture and asks such questions as ,What categories of experience are encoded by the members of this speech community through the linguistic choices that they make when they talk?"“ (Fillmore 1982: 111). Deppermann (2002: 
als lexikalische Semantik, wie sie traditionell durch Merkmalslisten erfasst wird, weshalb er auch von der Notwendigkeit einer „Verstehens-Semantik“ spricht (vgl. Fillmore 1985). ${ }^{5}$ Dieses Wissen ist strukturiert durch Frames.

By the term ,frame' I have in mind any system of concepts related in such a way that to understand any one of them you have to understand the whole structure in which it fits; when one of the things in such a structure is introduced into a text, or into a conversation, all of the others are automatically made available. (Fillmore 1982: 111)

Wörter verweisen nicht nur auf semantische und kognitive Kategorien, sondern sie ,evozieren' Frames, die komplexe Wissensbestände und Praktiken einer (Sprach-)Gemeinschaft umfassen (vgl. Fillmore 1982: 119). Die Kenntnis der Frames ist damit Voraussetzung für die Bedeutungskonstitution.

Während die Semantik von Verben in interaktionalen Ansätzen bisher äußerst wenig untersucht ist, bildet sie den Ausgangspunkt der Frame-Semantik Fillmorescher Prägung ${ }^{6}$ : Verben sind die am besten beschriebenen frame-evozierenden Elemente, weil sie durch ihre Argumentstellen am klarsten bestimmte Partizipanten in Sachverhalten (,Frame-Elemente') obligatorisch verlangen. Systematisch auf empirische Daten angewandt worden ist vor allem die durch das lexikographische Projekt FrameNet repräsentierte Version der Frame-Semantik (vgl. z. B. Fillmore/Johnson/Petruck 2003).

Für eine semantische Klassifikation von Korpusbelegen im Rahmen empirischer Untersuchungen (vgl. z. B. Zeschel/Proske 2015; Zeschel 2017) ${ }^{7}$ haben

19) weist aus gesprächsanalytischer Perspektive auf die Verwandtschaft ethnomethodologischer und kognitionswissenschaftlicher Konzepte der Rolle von Wissen bei der Bedeutungskonstitution hin: Aus wiederholter Erfahrung gewonnene, abstrahierende Muster bzw. verschiedene Wissensbestände (z. B. als Frames konzeptualisiert) können Interpretationen von Aktivitäten (einschließlich der Äußerung bestimmter sprachlicher Ausdrücke) leiten und diese wiederum „bestätigen“ zugleich die Gültigkeit der abstrakten Muster.

5 Die Betonung verstehenstheoretischer Aspekte findet sich vor allem in Fillmores Arbeiten der 80er Jahre. Später, vor allem im Rahmen von FrameNet, stehen eher praktische Aspekte der lexikographischen Erfassung der argumentstrukturellen Rahmen von Verben im Vordergrund (vgl. auch Busse 2012: 25).

6 Der kognitionswissenschaftliche Ansatz von Barsalou und auch viele linguistische Arbeiten widmen sich dagegen vor allem Frames, die von den Vertretern nominaler Wortarten ausgehen (vgl. a. Busse 2012: 21).

7 Für diese Studie zu kommen und gehen wurden Belege aus dem mündlichen Korpus FOLK und aus Schriftkorpora sowohl syntaktisch als auch semantisch klassifiziert, um quantifizierbare Aussagen über Form-Funktions-Korrelationen sowie präferierte Argumentrealisierungsmuster für eine Medialität, Gattung oder sequenzielle Handlung machen zu können. Da die semantische Klassifikation dabei nicht der eigentliche Fokus war, war keine möglichst detaillierte, sondern 
framebasierte Bedeutungsdefinitionen gegenüber Bedeutungsparaphrasen, wie sie sich in Wörterbüchern finden, den Vorteil, dass sie durch die Frame-Elemente weniger ambig sind als Formulierungen, die für die Partizipanten Pronomen wie jemand einsetzen. Durch die framespezifische semantische Spezifikation der Leerstellen wird eine zuverlässigere Klassifikation ermöglicht, auch wenn es natürlich weiterhin Uberlappungen zwischen verschiedenen Lesarten bzw. Frames polysemer Verben gibt. Ein weiterer Vorteil ist außerdem die vertikale und horizontale konzeptuelle Vernetzung der Frames untereinander: Dadurch ist zum einen das Granularitätsniveau für eine Untersuchung wählbar - man kann, wie in diesem Artikel, auf einer abstrakten Ebene bleiben und nur die jeweils hierarchiehöchsten Frames verwenden oder aber beliebig feine Subframes ansetzen - und zum anderen werden häufige Metaphorisierungsprozesse abgebildet, die für Untersuchungen zur Lexikalisierung neuer Bedeutungen wichtig sind (vgl. z. B. Sullivan 2013; zu Metaphern vgl. auch Schröder in diesem Band).

Auch wenn eine semantische Klassifikation von Korpusbelegen weitgehend introspektiv ist, kommen hier im Gegensatz zu Analysen von isolierten Einzelsätzen potenziell disambiguierende Kontextfaktoren ins Spiel; nicht realisierte Frame-Elemente können durch den sprachlichen oder außersprachlichen Kontext erschließbar sein. Insofern kann man hierbei prinzipiell zumindest teilweise der gesprächsanalytischen Orientierung an der Teilnehmerperspektive gerecht werden - die introspektiv entstandenen Frames können anhand von (Aufzeige-) Handlungen der Interaktionsteilnehmer plausibilisiert werden. Diese umfassen z. B. die Folgebehandlung eines zweiten Interaktionsteilnehmers allgemein, kollaborative Turnergänzungen und fremdinitiierte Reparaturen. Durch sie kann implizit oder explizit eine übereinstimmende Interpretation angezeigt werden.

Fälle der Plausibilisierung durch die Aufzeigehandlungen eines zweiten Sprechers sollen hier aber nicht an Beispielen diskutiert werden, denn zum einen gehören sie zu den allgemeinen Mechanismen der Bedeutungskonstitution, unabhängig von einer spezifischen Semantiktheorie, und zum anderen ist für bestimmte Untersuchungsgegenstände (wie die genannte exhaustive semantische Klassifikation von Belegen im Rahmen von Korpusuntersuchungen) eine semantische Analyse auch von Fällen, in denen keine Partnerhandlung die Interpretation stützt, notwendig. Jede systematische semantische Analyse stützt sich notwendigerweise auch auf reine Analytikerkategorien. Zudem kann auch die Analyse von Aufzeigehandlungen letztendlich keine Evidenz für die kognitive Realität eines Frames bieten, da eine solche Analyse verschiedene semantische

eine grobe, praktikable Frameanalyse angebracht. Schon diese jedoch warf einige grundsätzliche Fragen auf, die zu den hier dargelegten Überlegungen geführt haben. 
Ansätze - seien es kognitive Ansätze oder nicht - bestätigen kann; was auch immer man theoretisch vor der Analyse festlegt, lässt sich meist durch einzelne Beispiele bestätigen. Dennoch oder gerade deswegen ist eine linguistisch bzw. lexikologisch fundierte Systematisierung von Bedeutungen eines polysemen Lexems für bestimmte Fragestellungen eine Voraussetzung für interaktionale Analysen, weil eine solche Systematisierung allein durch interaktionale Analysen niemals erreicht werden kann.

Somit sind eine framesemantische Systematisierung von Bedeutungen und die Diskussion der einhergehenden Probleme natürlich auch nicht spezifisch für mündliche Daten. Vor allem Ambiguitäten zwischen Frames betreffen Schriftdaten gleichermaßen. Der vorliegende Beitrag will aber gerade überprüfen, inwiefern das Heranziehen von Gesprächsdaten und eine nur anhand dieser mögliche interaktionale Analyse, die die Introspektion ergänzt, einen Mehrwert bei der semantischen Analyse bringen kann und wo sie dabei an ihre Grenzen stößt. Die Spezifika der Spontansprache (Linearität, Inkrementalität, Sequenzialität, Dialogizität) erfordern zudem in jedem Fall zumindest die Reflexion dieser und der daraus resultierenden gesprächsanalytischen Methoden. Das heißt: Man kann mündliche Daten auch bei einer rein framesemantischen Analyse nicht einfach wie einen Text behandeln, ${ }^{8} \mathrm{da}$ ein solcher anderen Produktions- und Rezeptionsbedingungen unterliegt.

Im folgenden Abschnitt (3) wird exemplarisch anhand des Verbs kommen diskutiert, ob und inwiefern a) die Frame-Semantik geeignet ist, dessen Bedeu-

8 Dies tut Ziem (2015) über weite Strecken in seiner framesemantischen Analyse der Interpretation von Nullinstanziierungen in Talk-Show-Daten; er bezieht sequenzielle Aspekte nur sporadisch ein. Er geht davon aus, dass frameevozierende Elemente auch Konstitutenten vorausgehender Sätze (die teilweise viele Intonationsphrasen zurückliegen) rückwirkend FrameElement-Status zuschreiben können. Sicherlich lassen sich so nachträgliche Interpretationsprozesse (Kohärenzherstellung, Auflösen indirekter Anaphern) erklären, aber die Frage, welcher Frame durch eine solche Konstituente zum Zeitpunkt ihrer Produktion - bevor sie überhaupt zu dem folgenden Element in Bezug gesetzt werden kann, aufgerufen wird oder als Frame-Element welches anderen Frames sie bis dahin konzeptualisiert werden kann, wird nicht systematisch beantwortet. Bis auf ein Beispiel (vgl. Ziem 2015: 71) werden außerdem nur von Substantiven evozierte Frames - vollkommen unabhängig von den durch sie umgebende Verben evozierten Frames - betrachtet. Zwar beschränkt sich der vorliegende Beitrag umgekehrt auf verbale Frames, doch sind diese auch grundlegender, da sie in jedem Satz relevant sind, auch wenn er nur pronominal realisierte Argumente enthält. Substantive sind als Argumente immer auch von Verben abhängig, so dass es nicht ausreicht, die von ihnen evozierten Frames isoliert zu analysieren, zumindest nicht dann, wenn man die gesamte Bedeutung eines Turn(abschnitt)s erfassen und sich nicht auf die semantischen Relationen nominaler Elemente untereinander beschränken will. 
tungsspektrum zu erfassen, und b) sequenzanalytische Methoden introspektive semantische Urteile stützen können. Anschließend wird unter 4 erörtert, ob und inwiefern auch das Bedeutungs- und Funktionsspektrum der aus dem Imperativ komm entstandenen Partikel framesemantisch erfasst werden kann, ob sich also bei der Analyse von Synsemantika ein ähnliches oder anderes Verhältnis von gesprächsanalytischer und semantischer Methodologie zeigt.

\section{Framesemantische Analyse der Bedeutungen von kommen}

In framesemantischer Sichtweise sind nicht nur die Vernetzungen der verschiedenen Frames, die ein Wort evoziert, zu modellieren, sondern es gilt auch zu erfassen, wie ein mit einem Wort assoziierter Frame durch die Vernetzung mit anderen Wörtern mit ähnlicher, perspektivisch verschiedener oder komplementärer Bedeutung konstituiert wird (zu Perspektive und Perspektivierung vgl. auch Meier in diesem Band). ${ }^{9}$ Aus diesem Grund werden die Verben kommen und gehen häufig gemeinsam betrachtet (vgl. zu ihrem Gebrauch im gesprochenen Deutsch Proske 2016, 2017a, b; Zeschel 2017): Sie sind die unspezifischsten Bewegungsverben des Deutschen und beide hinsichtlich der Fortbewegungsart unterspezifiziert (sie können für Bewegungen zu Fuß oder mit einem Fahrzeug verwendet werden), unterscheiden sich aber zum einen durch die komplementäre Beobachterperspektive (kommen denotiert eine Bewegung auf den Beobachter zu, gehen eine von ihm weg) und zum anderen durch einige die Bewegungsart betreffende semantische Merkmale (vgl. Di Meola 1994): Die durch gehen bezeichnete Bewegung ist in der Regel eine intentionale, ungehinderte Eigenbewegung; kommen kann ebenfalls eine solche bezeichnen, im Gegensatz zu gehen aber auch Bewegungen, denen mindestens eins der drei Merkmale fehlt - vgl. z. B. Er ging $\left({ }^{*}\right.$ versehentlich) an den Tisch. vs. Er kam (versehentlich) an den Tisch.). Der kommenSatz hat eine Lesart, bei der zwei der Merkmale fehlen: Eine Bewegung, die zum

9 So instanziieren z. B. sowohl kaufen als auch verkaufen (neben anderen Verben) den Frame COMMERCIAL_TRANSACTION, evozieren aber jeweils einen diesen näher perspektivierenden Frame (COMMERCE_BuY und COMMERCE_SELL), da einmal der Käufer und einmal der Verkäufer Agens ist. COMMERCial_tRANSACTION besteht außerdem aus zwei Teilereignissen bzw. Subframes, COMMERCE_GOODS-TRANSFER und COMMERCE_MONEY-TRANSFER, die im Deutschen und Englischen nicht in unterschiedlichen Verben lexikalisiert sind, sondern z. B. in (ver)kaufen zusammenfallen, $d$. h. deren Bedeutungen umfassen beide Teilereignisse. $\mathrm{Zu}$ ähnlichen Relationen der Frames für kommen und gehen untereinander vgl. Fußnote 12. 
versehentlichen Berühren eines Gegenstandes führt, ist eine Eigenbewegung, aber sie ist nicht intentional und nicht ungehindert.

Darüber hinaus sind kommen und gehen auch in zahlreichen anderen, keine Bewegung denotierenden Lesarten lexikalisiert. In diesen haben sie in einigen Fällen auch eine andere Argumentstruktur - so kann z. B. nur ein einstelliges gehen, ohne das in Bewegungsbedeutung auftretende Direktionaladverbial, Bedeutungen wie ,etwas funktioniert' (Mein Handy geht wieder.) oder ,etwas ist akzeptabel" (Die Preise gehen eigentlich.) haben, und nur ein gehen mit expletivem Subjekt und einem zusätzlichen Dativargument und einem Modaladverbial kann die Bedeutung ,jemand befindet sich irgendwie' (Es geht mir gut.) haben. In anderen Fällen dagegen hat die Lesart eine Argumentstruktur, die auch mit Bewegungssemantik vorkommt. So tritt kommen in der Lesart ,etwas hat seinen Ursprung irgendwo' immer mit einem Quell-Direktional auf (Diese Geschichte kommt aus dem Mittelalter.), wie es auch bei Bewegungslesart des Verbs möglich ist (Maria kommt aus dem Garten.).

Wenn man sich authentische Verwendungsbeispiele ansieht, stellt sich im Hinblick auf die Zusammenhänge von Argumentstruktur und Lesart folgende Frage: Wie können Sprecher und Rezipienten verschiedene Lesarten mit derselben Argumentstruktur identifizieren bzw. auseinanderhalten? Dies ist nicht nur für die genannten Fälle mit formal gleichen obligatorischen Argumenten (vgl. kommen aus) relevant, sondern auch für Fälle, bei denen ein eigentlich obligatorisches, konzeptuell notwendiges Argument weggelassen wird und so formal ein für eine andere Lesart typisches Argumentrealisierungsmuster entsteht. An Beispielen soll gezeigt werden, dass die nicht realisierten Frame-Elemente meist kontextuell erschließbar sind und außerdem die deiktischen Merkmale von kommen und gehen Hinweise auf das zu rekonstruierende Frame-Element geben, dass es aber in Fällen einander überlappender, hierarchisch voneinander abhängiger Frames häufig vorkommt, dass man den Frame bzw. das Granularitätsniveau ${ }^{10}$ nur introspektiv bestimmen kann, da weder kontextuelle Faktoren noch die deiktischen Merkmale disambiguierend wirken. Zunächst sollen jedoch die für die Analysen relevantesten Frames - zitiert nach FrameNet ${ }^{11}$ - eingeführt werden (weniger zentrale werden im Fließtext in Klammern angegeben):

- Motion: Some entity (Theme) starts out in one place (Source) and ends up in some other place (Goal), having covered some space between the two (Path).

10 So kann man z. B. alle Belege von kommen und gehen mit Bewegungsbedeutung unter dem abstrakten MoTiON-Frame erfassen oder aber feinkörniger vorgehen und sie verschiedenen, die Bewegungsart, -richtung usw. spezifizierenden Subframes zuordnen (vgl. Fußnote 12).

11 Vgl. http://framenet.icsi.berkeley.edu/ [15.11.2017] 
Alternatively, the Area or Direction in which the Theme moves or the Distance of the movement may be mentioned. ${ }^{12}$

- TEMPORARY_STAY: A Guest stays for a time at a Location, which is not his or her permanent residence, for some Duration. The Location is often the permanent residence of a Host.

- CAUSE_motion: An Agent causes a Theme to undergo translational motion. Although different members of the frame have different degrees of profiling of the trajectory, the motion may always be described with respect to a Source, Path and/or Goal. ${ }^{13}$

In Beispiel (1) finden sich in Z. 8 und 9 Verwendungen, bei denen das fehlende Frame-Element (das Ziel auf meinen Geburtstag), direkt vorerwähnt ist (Z. 6), so dass es plausibel ist, für diese dieselbe Lesart wie für die Verwendung in Z. 6 anzunehmen (und nicht etwa eine nur mit einstelligem kommen mögliche ${ }^{14}$ ).

12 In FrameNet werden zahlreiche Subframes für MOTION angeführt, unter anderem ARRIVING (,An object (Theme) moves in the direction of a Goal. The Goal may be expressed or it may be understood from context, but it is always implied by the verb itself.'), DEPARTING (,An object (the Theme) moves away from a Source. The Source may be expressed or it may be understood from context, but its existence is always implied by the departing word itself.') und SELF_MOTION (,The Self_mover, a living being, moves under its own direction along a Path. $\therefore$ Diese stellen verbspezifische Perspektivierungen des verbübergreifenden Frames dar, sie werden hier als semantische Merkmale von kommen (Bewegung vom Beobachter weg) und gehen (Bewegung auf den Beobachter zu; in der Regel intentionale, ungehinderte Eigenbewegung) betrachtet (vgl. Di Meola 1994), was nicht dagegen spricht, sie ebenfalls als Frames zu modellieren. Da die genannten Merkmale jedoch zusätzlich zum Frame - zumindest bei vielen Frames, die die Verben evozieren können, - evoziert werden, müsste man eine Frame-Kombination annehmen, und zu diesem Zweck müssten die Merkmale von ARRIVING und DEPARTING abstrakter gehalten werden, um auch für Kombinationen mit metaphorischen Lesarten offen zu sein. Für die hier geführte Argumentation sind solche Details hinsichtlich der Granularität jedoch nicht unmittelbar relevant. 13 Auch CAUSE_motion hat einige Subframes (wie PlaCing und Removing), die den jeweils bezeichneten Sachverhalt genauer perspektivieren, die in ihrer hohen Granularität hier aber nicht relevant sind.

14 Z. B. ,sich ereignen' (Die schwerste Prüfung kommt noch.) oder, einen Orgasmus haben“ (Sie ist noch nicht gekommen.). 
(1) Tischgespräch, FOLK_E_00047_SE_01_T_02, c222, 00:37:23.22 ${ }^{15}$

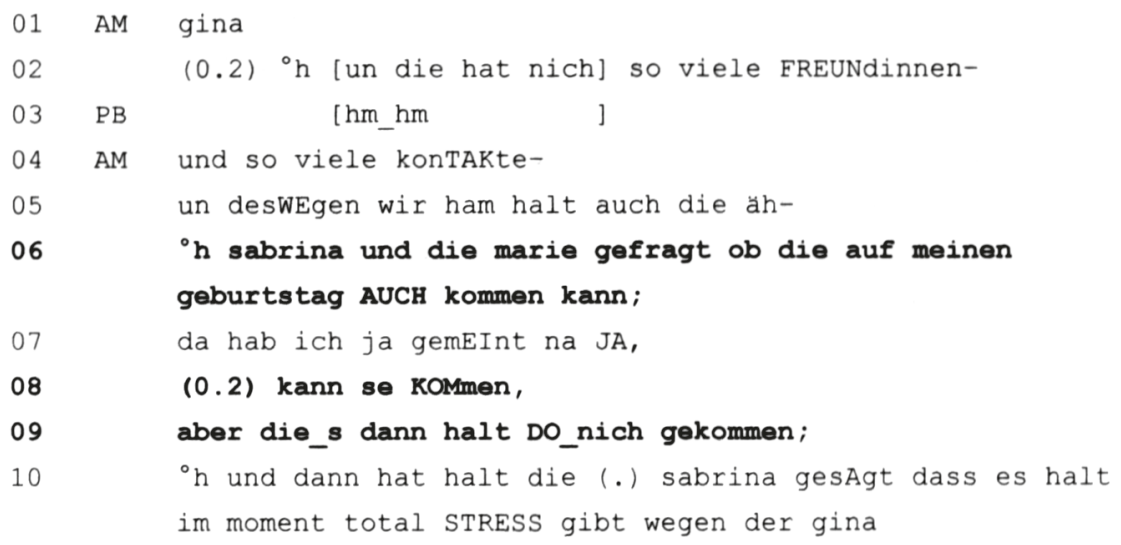

Eine nicht über den Kontext, sondern nur introspektiv-analytisch lösbare Frage ist dagegen, welcher Frame - von allen, die ein (konkretes oder abstraktes) Ziel enthalten - hier überhaupt angesetzt werden soll. Man kann sowohl den Motion-Frame als auch den Frame Participation (,An Event with multiple Participants takes place. [...]') ansetzen, bei dem das abstrakte Ziel als Ereignis (Geburtstag(sfeier)) und das abstrakte Thema als Teilnehmer (Gina) an diesem konstruiert wird. Die beiden Frames können zudem entweder als metaphorisch oder metonymisch miteinander verbunden angesehen werden (Teilnahme als Bewegung konzeptualisiert oder Bewegung zum Ereignisort als Teilhandlung eines Ereignisses, das auch (mindestens) eine Handlung am Ereignisort umfasst bzw. impliziert). Die Entscheidung hängt nicht zuletzt davon ab, auf welchem Granularitätsniveau man die Analyse aus theoretischen Gründen überhaupt durchführen möchte, $d$. h. welche Auswahl an Frames man einbezieht. Die vorliegende Analyse kann nur versuchen, die Sicht der Sprecherin zu rekonstruieren - es ist wahrscheinlich, dass sie ihre selbst gebrauchte Lesart wieder verwendet; das Verstehen des Rezipienten ist in diesem Beispiel nicht anhand von Displays ablesbar. Das Fehlen einer Reaktion legt zumindest nah, dass er den Turn der Sprecherin verstanden hat bzw. interpretieren konnte. Die mentale Evokation eines Frames, der schon in Bezug auf die Sprecherin selbst den Annahmen des Analytikers entspringt, lässt sich damit aber natürlich nicht belegen.

15 Alle Beispiele stammen aus dem Forschungs- und Lehrkorpus gesprochenes Deutsch (FOLK) (http://agd.ids-mannheim.de/folk.shtml [15.11.2017]) und sind nach GAT 2 (vgl. Selting et al. 2009) transkribiert. 
In Beispiel (2) ist das nicht realisierte Ziel von kommen in Z. 16 nicht vorerwähnt, kann aber aus dem Kontext inferiert werden: AM berichtet davon, dass eine Bekannte ihren Freund nur selten sehe, unter anderem weil er häufig lieber etwas mit seinen Freunden unternehme, und dass die beiden nicht in der Wohnung des jeweils anderen übernachteten, obwohl sie mittlerweile in derselben Stadt wohnten. Und wenn er einmal komme, sei er blöd zu ihr. In diesem Zusammenhang kann das nicht realisierte Ziel von kommen nur die Wohnung der Bekannten sein. Auch hier stellt sich allerdings wieder die Frage, welcher Frame überhaupt vorliegt, da neben dem MoTION-Frame auch andere, z. B. TEMPORARY_STAY oder VISITING, ansetzbar sind. ${ }^{16}$

(2) Tischgespräch, FOLK_E_00047_SE_01_T_02, c259, 00:38:19.62

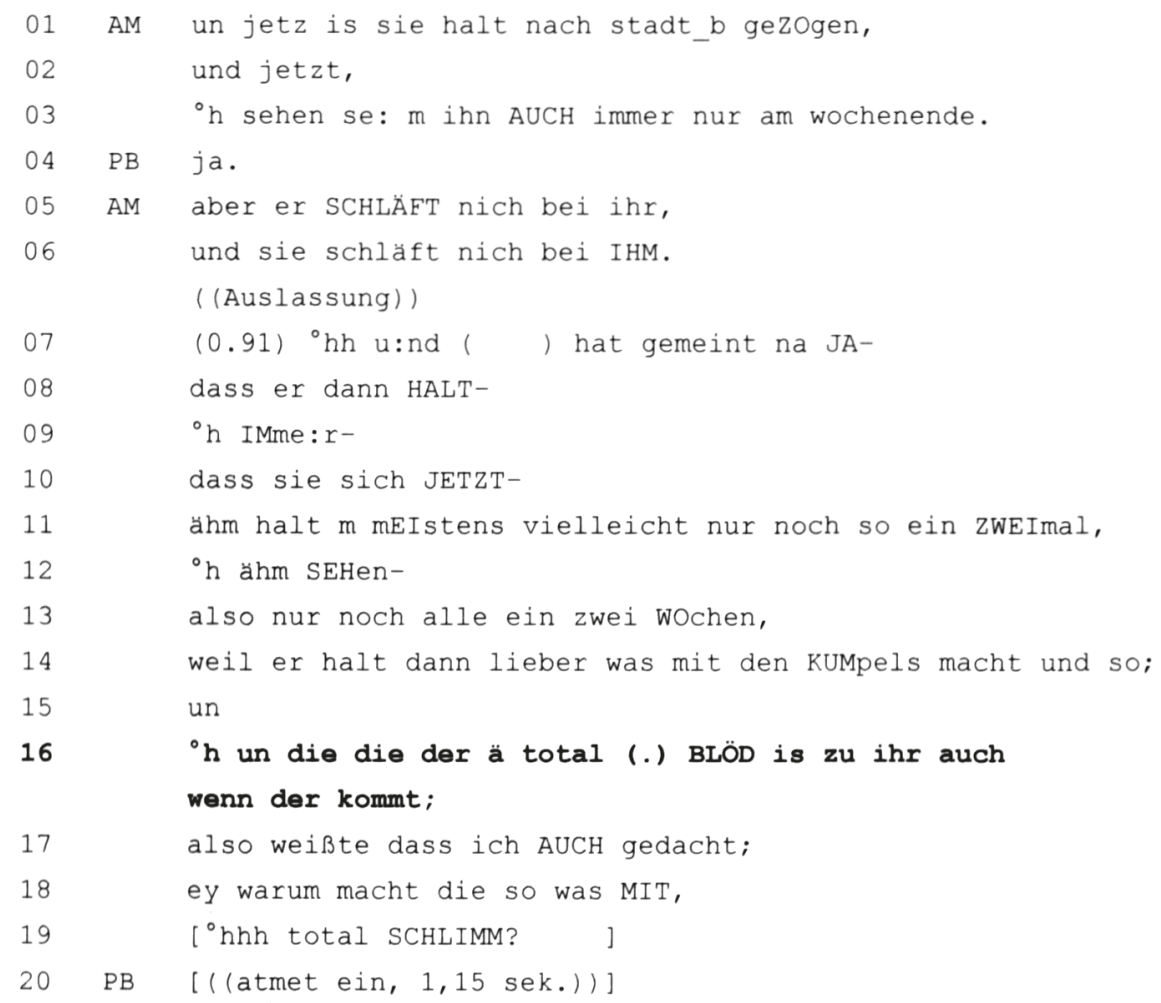

16 Zur Definition von TEMPORARY_STAY siehe oben, zu einem weiteren Beispiel (4) weiter unten. VISITING wird in FrameNet folgendermaßen definiert: ,An Agent matches location with an Entity in order to fulfill some Purpose. Quite often, the Purpose is social- or entertainment-oriented.‘. 
Beispiel (3) zeigt, dass es nicht nur vom sprachlichen Kontext, sondern auch von verschiedensten anderen Teilen des common ground (vgl. z. B. Clark 1996) ${ }^{17}$ abhängen kann, welcher Frame evoziert wird. Dem Satz Dann kommt ihr ins Museum. (Z. 15) kann, isoliert präsentiert oder für nicht über genug Kontextwissen verfügende Zuhörer, sowohl MoTION als auch CAUSE_MOTION zugeschrieben werden; es ist nur über Inferenzen erschließbar, dass im vorliegenden Kontext letzterer Frame evoziert wird.

(3) Tischgespräch, FOLK_E_00053_SE_01_T_01, c633, 00:16:49.83

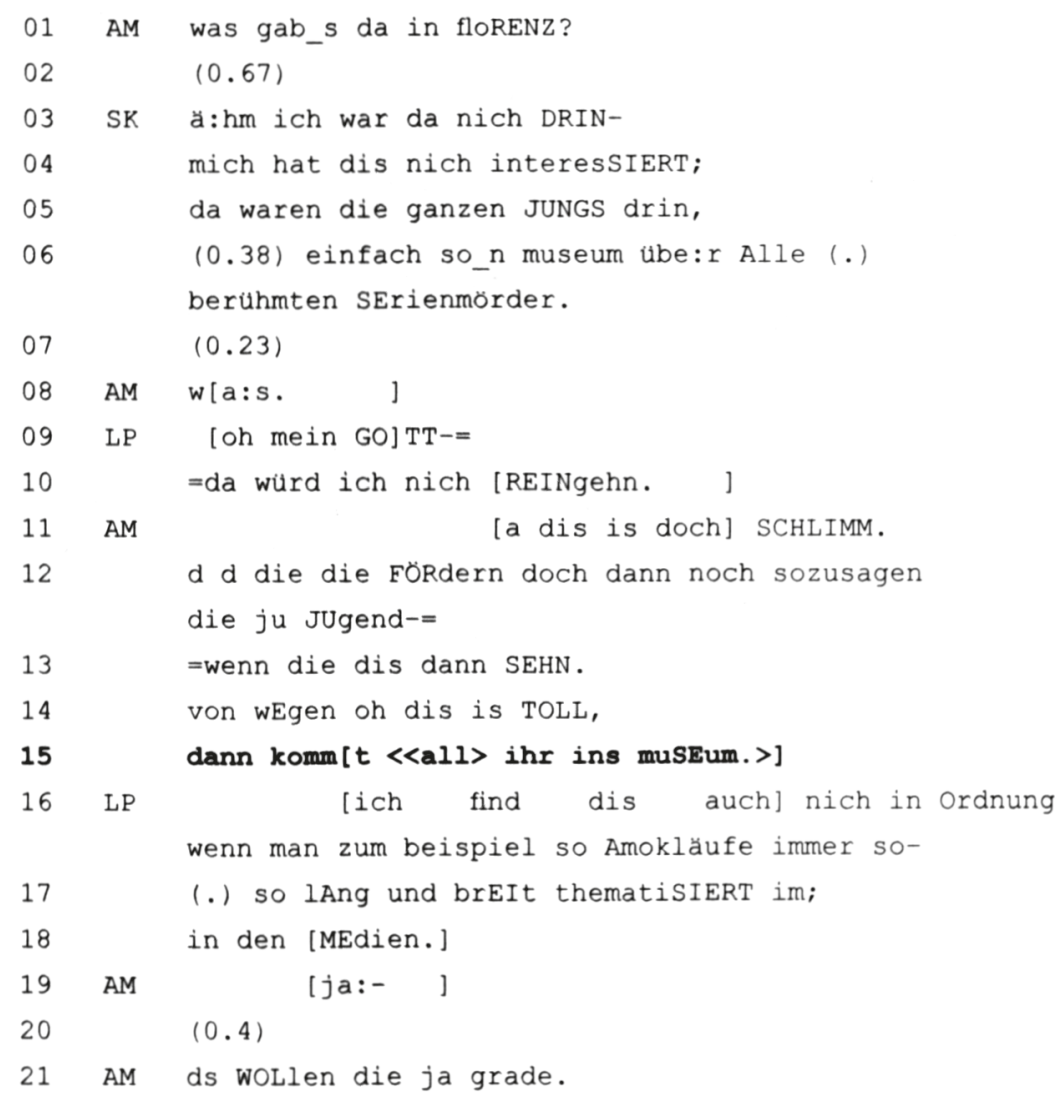

17 Frames sind größtenteils Teil des communal common ground im Sinne Clarks (1996), also von ganzen sozialen Gruppen geteilten Wissensbeständen, im Unterschied zu nur mit bestimmten anderen geteilten, die Interaktionsgeschichte betreffenden Teilen (personal common ground). 
AM begründet ihre Meinung, dass ein Museum über Serienmörder schlimm sei ( $Z$. 11), damit, dass die Sichtbarkeit von Mördern bzw. ihren Geschichten bestimmte Ansichten der Jugend fördere (Z. 12-13), und illustriert ihre Vorstellung der Wirkung auf Jugendliche mit einer durch von wegen (Z. 14; vgl. Bücker 2008) eingeleiteten, an diese gerichteten, fiktiven wörtlichen Rede, die den interessierenden Satz enthält. Dessen Frame wird weder allein von der realisierten Argumentstruktur evoziert - da diese mit verschiedenen Frames auftritt -, noch trägt die Referenz der Argumente dazu bei, da diese eindeutig und für beide Frames dieselbe ist. Für die Interpretation entscheidend ist das dann (vgl. Deppermann/Helmer 2013), das auf die Bedingung ( $=$,wenn ihr Serienmörder seid') für den Sachverhalt ,ins Museum kommen' Bezug nimmt, und diese ist nicht dem Kontext zu entnehmen, sondern muss über eine längere Schlussfolgerungskette, die auf communal common ground (vgl. Clark 1996: 100-112) angewiesen ist, inferiert werden. Die Schlussfolgerungskette lässt sich folgendermaßen zusammenfassen: Taten, für die man in die Geschichte eingeht, sind interessant (bzw. toll). Darüber hinaus ist es erstrebenswert, sich nicht nur über für geschichtsträchtige Taten bekannte Personen zu informieren, indem man ins Museum geht - das wäre die andere, auf Eigenbewegung hinauslaufende Lesart von Dann kommt ihr ins Museum (und guckt euch die Akteure dieser Geschichten an) ${ }^{18}$-, sondern selbst durch entsprechende Taten den Status zu erreichen, einen Platz im Museum zu bekommen, also metonymisch dorthin bewegt zu werden (nicht die Person selbst, sondern mit ihr verbundene Gegenstände und Beschreibungen ihrer Geschichte werden ausgestellt). Auch der verbreitete Topos, dass die (mediale) Thematisierung von Verbrechen zum Verüben solcher anstifte, wird vor dem kommen-Satz an keiner Stelle expliziert. ${ }^{19}$ Ohne das entsprechende Hintergrundwissen ist die hier intendierte Bedeutung des Verbs nicht erschließbar.

18 Selbst wenn diese unwahrscheinlich ist, weil eine Perspektivierung vom Zielort aus, die mit kommen in Bewegungsbedeutung einhergeht, hier weniger plausibel erschiene als eine Verwendung des quellperspektivierenden gehen mit Bewegungsbedeutung, und so keine echte Disambiguierung nötig ist, ist das Verstehen der Verwendung von CAUSE_MOTION auf nicht explizierten communal common ground angewiesen.

19 Erst Z. 16 bis 21 lassen sich als zumindest einen Teil dessen, was diesen Topos ausmacht, andeutend interpretieren: Als Zweitbewertung zu AMs Ansicht äußert LP, dass Medienberichte über Amokläufe ebenso abzulehnen seien wie das thematisierte Museum. AM bestätigt dies und begründet ihre Bestätigung damit, dass Amokläufer sich Medienpräsenz wünschen. Sie sagt zwar nicht, dass sie oder andere Beobachter diese Medienpräsenz als potenziell zur Nachahmung anstiftend betrachten, die Möglichkeit einer entsprechenden Inferenz ergibt sich jedoch aus der vorausgehenden Gleichsetzung von Museums- und Medienpräsenz und dem von AM zuvor vermuteten Nachahmungswunsch durch Museumspräsenz. 
Die folgenden beiden Beispiele zeigen, wie derselbe Frame einmal kontextfrei identifizierbar ist, weil ein konzeptuell obligatorisches Frame-Element, das in keinem anderen Frame mit kommen möglich ist, realisiert ist, und einmal nicht kontextfrei identifiziert werden kann, sondern über den Kontext mit evoziert werden muss, weil es nicht realisiert ist. Der Satz in (4), Z. 5 ist auch isoliert dem Frame TEMPORARY_STAY (vgl. oben) zuordenbar, weil das durative Temporaladverbial bzw. das Frame-Element Duration durch für ein oder zwei Stunden realisiert ist. Das Frame-Element Location ist zwar nicht realisiert, ist aber nicht konstitutiv für die Bedeutung, weil der Ort immer zumindest kontextuell gegeben ist; zudem ist Location kein für diesen Frame spezifisches Element, d. h., andere Frames mit kommen enthalten ebenfalls eines im Kern- oder Peripheriebereich. ${ }^{20}$

(4) Tischgespräch, FOLK_E_00055_SE_01_T_02, c1, 00:00:00.00

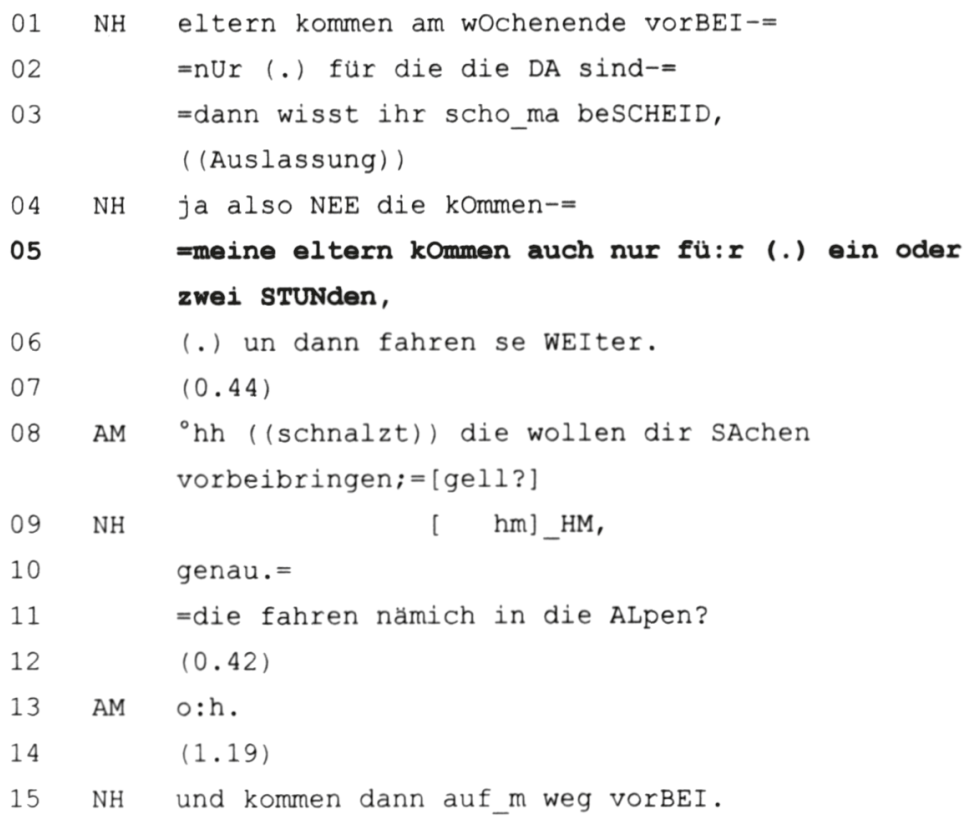

20 Der Frame HAVING_oR_LACKING_ACCESS enthält z. B. ein solches Element: ,A Theme has access to a Useful_location or is blocked from it by a Barrier. (Mit diesem Schlüssel kommt man in die Abstellkammer). 
Das durative Temporaladverbial unterscheidet den in Z. 5 evozierten Frame deutlich von dem in Z. 1 und 15 (durch vorbeikommen) evozierten: In Z. 1 informiert NH ihre WG-Mitbewohnerinnen zunächst darüber, dass ihre Eltern sie besuchen werden, das Temporaladverbial am Wochenende ist nicht durativ; man kann hier den Motion-Frame ansetzen. ${ }^{21}$ Mit der Verwendung von kommen im Frame TEMPORARY_STAY in Z. 5 dagegen spezifiziert sie die Dauer des Aufenthalts. In Z. 15 wird der Zeitpunkt des Besuchs noch einmal spezifiziert, es liegt derselbe Frame vor wie in Z. 1.

Der von dem Satz in (5), Z. 16 evozierte Frame ist dagegen nicht kontextfrei identifizierbar. Man würde ihn isoliert dem MотіоN-Frame zuordnen, weil er ein Zieladverbial, aber kein duratives Temporaladverbial enthält. Auch der mit ihm koordinierte Folgesatz (Z. 17) bietet keine hinreichenden Hinweise auf eine andere Semantik. Dennoch ist durch den gesamten vorausgehenden Kontext klar, dass kommen hier nicht einfach auf Bewegung, sondern auf einen Aufenthalt verweist:

(5) Meeting, FOLK_E_00026_SE_01_T_03, c2172, 02:49:58.95

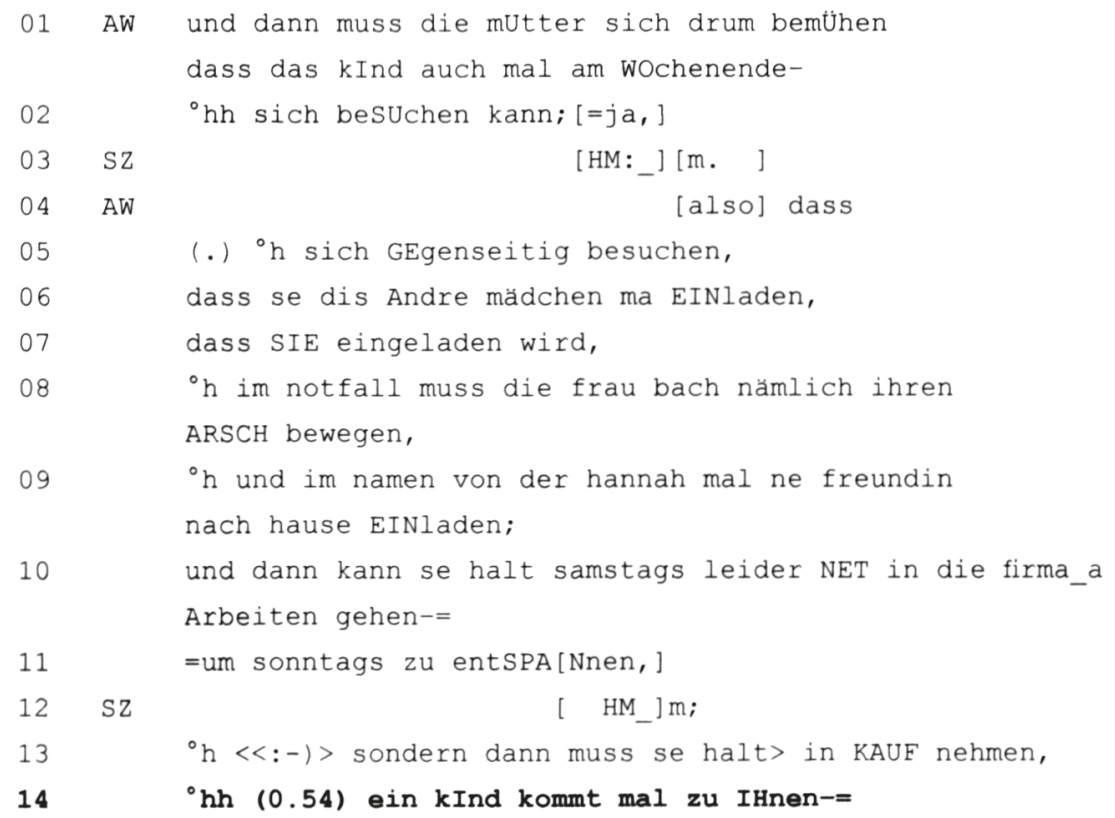

21 Je nach Granularitätsniveau kann auch der Frame VISITING (vgl. Fußnote 16) angesetzt werden. 
AW, Mitarbeiterin einer sozialen Einrichtung, äußert bei einem Meeting die Meinung, dass die Mutter eines Mädchens sich um die Möglichkeit von Besuchen (Z. 2 und 5) und Einladungen (Z. 6, 7 und 9) von Freundinnen der Tochter kümmern müsse. Dabei müsse sie mit einer Einschränkung ihrer eigenen Pläne und Vorlieben rechnen (Z. 10-11). Der hier semantisch zu diskutierende Satz ist in einen Matrixsatz (Z. 13) eingebettet, der den Sachverhalt Ein Kind kommt mal zu Ihnen und es gibt noch mehr Radau. als etwas, das man in Kauf nehmen muss, beschreibt. ${ }^{22}$ Das im zweiten Teilsatz genannte Ereignis (Radau) bezieht sich nicht auf eine Bewegung oder den Moment einer Ankunft, sondern auf eine längere Zeitspanne, einen Aufenthalt.

Als Fazit zu diesem Abschnitt lässt sich zunächst festhalten, dass eine framesemantische Beschreibung authentischer Gesprächsdaten sowohl kognitiv als auch sequenzanalytisch plausible Ergebnisse liefert und dabei den Vorteil hat, eine Vernetzung möglicher Lesarten und Ambiguitäten zur Verfügung zu stellen sowie durch die Annahme über die traditionellen Satzglieder hinausgehender Frame-Elemente eine differenziertere Lesartenidentifikation und -klassifikation zu ermöglichen. Die Analysen zeigen außerdem, dass oft Kontext für eine Disambiguierung notwendig ist und dass die Frame-Evokation bzw. Bedeutungskonstitution damit über den Satzrahmen hinausgeht.

Während die Frame-Semantik also bei der Analyse von Autosemantika in interaktionalen Daten eine methodisch nicht von der Gesprächsanalyse leistbare Rolle einnehmen kann, soll Abschnitt 4 zeigen, dass sie bei der Analyse der Bedeutung und Funktion von Synsemantika einen geringeren Mehrwert hat. Deshalb wird sie bei der Bedeutungs- und Funktionsanalyse dort zunächst nicht vorausgesetzt. Der als Partikel grammatikalisierte Imperativ komm lässt sich anhand sequenzanalytischer Methoden - unter Hinzunahme allgemeinen, relativ theorieneutralen linguistischen Wissens zum Herkunftsverb kommen - hinreichend beschreiben. Es wird gezeigt, dass eine kognitive, framesemantische Sicht

22 Statt TEMPORARY_STAY könnte auch hier der allgemeinere, diesem übergeordnete Frame VISITING angesetzt werden (vgl. Fußnote 16), u. a. weil dieser Frame das hier nicht realisierte Frame-Element Duration im Gegensatz zu TEMPORARY_STAY nicht braucht, so dass auch eine kontextfreie Identifikation denkbar wäre. Allerdings ist das argumentstrukturelle Muster jemand kommt zu jemandem konventionell nicht nur mit VISITING, sondern auch mit MOTION assoziiert, so dass es eine weitere potenzielle Ambiguität gibt. Die obige Argumentation schließt MOTION als in (5) relevanten Frame aus, weil die Interpretation als Aufenthaltslesart (VISITING oder TEMPORARY_STAY) plausibler erscheint. 
auf den semantischen Teil des linguistischen Wissens keinen Vorteil bringt, weil entscheidende Bedeutungsbestandteile durch eine solche gerade nicht erklärt werden können und die Evokation eines Frames durch Partikeln an sich wenig plausibel ist. Zwar ließe sich aus framesemantischer Sicht davon ausgehen, dass im Verlauf der Grammatikalisierung der Partikel mindestens einer der vom Verb kommen evozierten Frames eine Rolle für deren Bedeutungsentwicklung gespielt hat, doch ein für die synchrone Verwendung adäquater Frame müsste sehr abstrakt sein. Es stellt sich z. B. die Frage, ob trotz des bei der Partikel nie realisierten Ziel-Adverbials ein Frame-Element Goal (oder etwas entsprechendes Abstraktes) angesetzt werden kann.

\section{Bedeutungskonstitution beim Partikelgebrauch - am Beispiel von komm}

Bei der Bestimmung der Bedeutung von Partikeln wird in der linguistischen Literatur häufig ebenso introspektiv vorgegangen wie bei der Bestimmung von in einen Satz eingebetteten Wörtern. So werden z. B. oft Bedeutungen für Interjektionen ohne Kontext aufgelistet oder mögliche (Satz-)Kontexte erfunden (vgl. Nübling 2004: 15). In der Gesprächsanalyse dagegen werden die Funktionen einzelner Partikeln aus detaillierten Sequenzanalysen abgeleitet (vgl. z. B. Heritage 1984); auf semantische Ansätze wird dabei in der Regel aber nicht rekurriert. Dies mag in vielen Fällen auch nicht unmittelbar nötig sein, kann aber in Fällen, in denen die Partikel auf Autosemantika zurückgeht, die Analyse bereichern. Im Folgenden soll am Beispiel der Diskurspartikel komm gezeigt werden, dass eine Kombination interaktionslinguistischer und semantischer Analysen am geeignetsten ist, um sowohl die kontextabhängigen als auch die einzelfallübergreifenden Aspekte der Bedeutungskonstitution zu erfassen. Die semantische Analyse muss dabei aber nicht kognitiver bzw. framesemantischer Art sein.

Die deimperativische Partikel komm wird in der Literatur den sekundären Interjektionen oder den Diskursmarkern zugeordnet, die Angaben $\mathrm{zu}$ ihrer Semantik und Funktion reichen dabei von ,Aufforderung zu einem bestimmten Verhalten' (Wahrig-Burfeind 2012: 1923) über den Ausdruck von ,Kritik‘, ,Beschwichtigung“ (Schumacher et al. 2004: 498), ,Abwehr', ,Ungläubigkeit‘ und ,Zweifel“ (Reisigl 1999: 225) bis hin zu ,Konzessivität‘ (vgl. Auer/Günthner 2005: 346). $\mathrm{Zu}$ diesen Zuschreibungen gelangen die Autoren in der Regel über introspektiv analysierte, ausgedachte Einzelsätze, denen komm vorangestellt ist; bei 
Auer/Günthner wird ein einzelnes authentisches Gesprächsbeispiel analysiert. ${ }^{23}$ Die Analyse einer Kollektion von 165 Beispielen aus FOLK (vgl. hierzu ausführlich Proske 2014) hat gezeigt, dass sich eine unterspezifizierte Bedeutung bzw. Funktion (,Aufforderung zum Aktivitätswechsel mit Appell an den common ground‘) für alle Fälle ansetzen lässt; die in der bisherigen Literatur angegebenen, spezifischeren Bedeutungen dagegen treffen nie auf alle Fälle und auch nicht auf ein formal eingrenzbares Subset der Fälle zu, sondern ergeben sich aus kontextuellen Faktoren - der vorausgehenden Interaktion oder anderem als geteilt unterstelltem Wissen oder aber der Folgeäußerung. Dies lässt sich zunächst sequenzanalytisch feststellen, also ohne bestimmte semantiktheoretische Annahmen, ebenso wie das funktionale Spektrum, das sich aus der unterspezifizierten Bedeutung ergibt.

Der Aktivitätswechsel, zu dem durch komm aufgefordert wird, umfasst immer das Beenden einer vorausgehenden und den Beginn einer erwarteten folgenden Aktivität; oft ist dabei aber nur eines von beidem kontextuell salient. Im folgenden Beispiel (6), Z. 09 ist es der Beginn einer neuen Aktivität; zudem macht hier der vorangehende und folgende Imperativ (nimm ihn) explizit, zu welcher Handlung aufgefordert wird - im Rahmen eines Fußballmanagerspiels fordert PL CH dazu auf, den von ihm vorgeschlagenen Spieler gleich mit dem zugehörigen Einstiegsgebot zu kaufen.

(6) Spielinteraktion, FOLK_E_00021_SE_01_T_01, c783, 00:09:56.14

$\begin{array}{lll}01 & \text { CH } & \text { ja oKAY }= \\ 02 & & =\text { dann schlag ich den CAio vor; } \\ 03 & & \text { von FRANKfurt. } \\ 04 & \text { XM1 } & <<p>\text { och }> \\ 05 & \text { CH } & \text { zWEI millionen MITtelf } \\ 06 & \text { XM1 } & <<p>\text { och }> \\ 07 & & (0.26) \\ 08 & \text { PL } & \text { NIMM ihn. } \\ 09 & & \text { komm NIMM ihn. } \\ 10 & \text { DK } & \text { hm. }\end{array}$

23 Dies ist untypisch für das Vorgehen der Interaktionalen Linguistik und der Tatsache geschuldet, dass komm nur in einem Überblicksartikel zu Diskursmarkern behandelt wird. Üblicherweise liegen den Analysen der Funktionen von Diskursmarkern - wie z. B. weil und obwohl - Kollektionen von authentischen Beispielen zugrunde (vgl. z. B. Gohl/Günthner 1999; Günthner 1999). 
$\begin{array}{lll}11 & \text { CH } & \text { echt? } \\ 12 & & (0.31) \\ 13 & \text { Sk } & \text { nó moME:NT. }\end{array}$

Komm fungiert hier nicht nur als ,Illokutionsverstärker bzw. impliziert Ermunterung, sondern verweist zugleich darauf, dass es als common ground gelten kann, dass niemand mehr für den unbekannten Spieler (die Unbekanntheit lässt sich dem größeren, hier nicht enthaltenen Transkriptkontext entnehmen) bieten wird (obwohl dies in der Folge doch noch geschieht), so dass eventuelle gedankliche Abwägungen von Handlungsalternativen beendet werden können.

In Beispiel (7) dagegen ist nicht aus den unmittelbar vorangehenden oder folgenden Turn-Bestandteilen klar, zu was aufgefordert wird, es lässt sich aber aus dem sequenziellen Kontext (also dem personal common ground (Clark 1996: 100-112) und zu ziehenden Inferenzen) erschließen:

(7) Berufsschule, FOLK_E_00008_SE_01_T_01, c995, 00:25:34.03

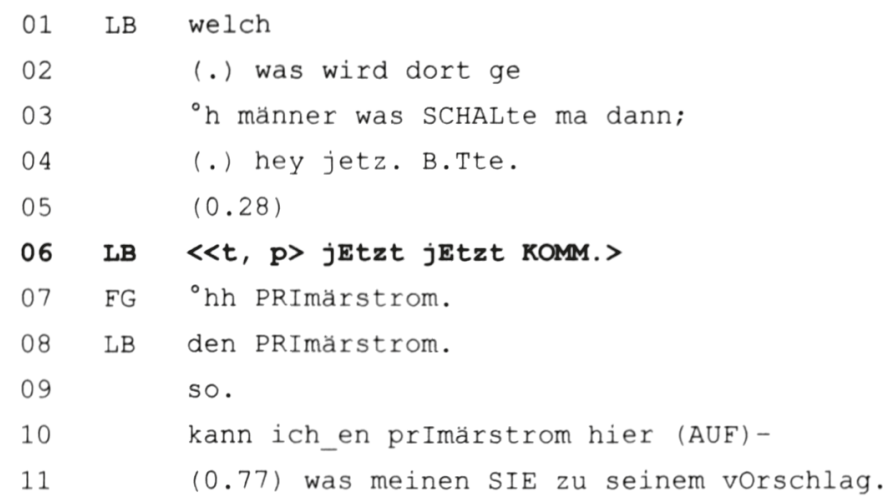

Der Lehrer (LB) hat eine Frage schon mehrfach gestellt, aber bisher nur Antworten bekommen, die diese nicht direkt beantworten, so dass er noch einmal fragt (Z. 01-03), gefolgt von Dringlichkeit signalisierenden Partikeln und Adverbien (Z. 04). Nachdem eine Antwort ausbleibt (Z. 05), reaktualisiert er die konditionelle Relevanz noch einmal durch ein leises, tiefer gesprochenes jEtzt jEtzt KOMM. (Z. 06) Durch komm fordert er die Schüler dazu auf, die nächste relevante Handlung auszuführen; diese muss nicht expliziert werden, da sie inferiert werden kann - der sequenzielle Kontext projiziert eine Antwort, und der größere interaktionsgeschichtliche Kontext enthält die dafür nötige Information. Auch die dann doch folgende Antwort (Z. 07) zeigt, dass die auf eine Wissensexplikation oder 
Transferleistung abzielende Frage es als dispräferiert erscheinen lässt, statt einer Antwort auf Nicht-Wissen zu verweisen.

In Beispiel (8) wird der erste Teil des Aktivitätswechsels fokussiert, es wird zum Beenden bzw. Ablassen von einer Handlung aufgefordert: SK fragt beim Monopolyspielen, ob sie bei ihrem nächsten Spielzug einen Hunderter wechseln könne (Z. 03), worauf ihr Vater (VK) mit ach KOMM; is doch eGAL. (Z. 04-05) reagiert.

(8) Spielinteraktion, FOLK_E_00011_SE_01_T_06, c109, 00:33:12.82

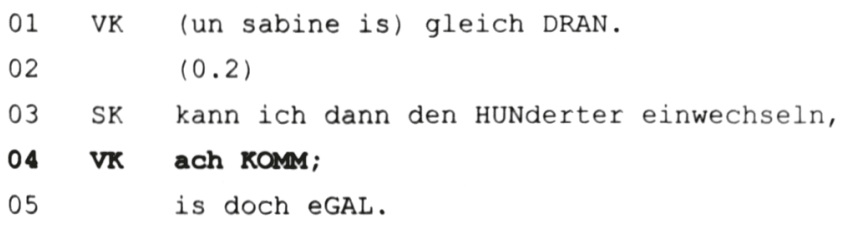

Da dem komm hier keine Aufforderung folgt und es auch keine nächste relevante Handlung gibt, die SK umsetzen müsste, sind weitreichendere Inferenzen nötig, um VKs Turn zu verstehen; diese beziehen sich nicht auf den sequenziellen oder interaktionsgeschichtlichen Kontext, sondern zum einen auf Wissen um Spielregeln und angemessenes Spielverhalten und zum anderen auf sprachliches Wissen um die Bedeutung von komm. Die der Partikel folgende Bewertung macht zwar ebenso wie das vorausgehende ach klar, dass hier eine Annahme abgelehnt wird, doch um darauf schließen zu können, zu welcher Handlung bzw. zum Ablassen von welcher Handlung damit implizit aufgefordert wird, muss man zum einen wissen, dass es zwar möglich, aber nicht nötig, sondern unter Umständen zeitraubend ist, große Spielgeldscheine gegen kleinere einzutauschen, wenn man keinen Zahlungsbedarf hat. Zum anderen muss man die neben der unterspezifizierten Bedeutung von komm lexikalisierte bewertende Bedeutung der Partikel kennen. Diese lässt sich paraphrasieren als ,ach Quatsch' oder ,sei vernünftig، (vgl. zur Annahme dieser Bedeutung im Detail Proske 2014). Zwar kann argumentiert werden, dass sich die bewertende Komponente aus dem Folgesatz ergibt und für die Partikel die o.g. unterspezifizierte Bedeutung vorliegt, doch könnte im vorliegenden Beispiel ach komm auch allein stehen; auch in diesem Fall wäre keine nicht-bewertende Lesart möglich.

Bezieht man bei der induktiven, sequenziellen Erschließung einer einzelfallübergreifenden Bedeutung nun die deiktischen Bedeutungsanteile des Herkunftsverbs kommen ein, kann man als weitere konstante Komponente Folgendes hinzufügen: Es liegt immer ein gegebenes, vom Beobachterstandpunkt aus 
identifizierbares (abstraktes) Ziel vor; es ist also kein Zufall, dass komm auf eine nächste relevante, sich aus dem common ground ergebende Handlung verweist. Dieses wird allerdings nie syntaktisch (als Direktionaladverbial) realisiert, es handelt sich also um eine rein semantische Komponente. Die Verbindung zum Autosemantikum sorgt für eine diachron plausible und außerdem systematische Bedeutungszuschreibung. Sie ist aber für das Feststellen weiterer hinzukommender Bedeutungsaspekte, wie sie sich in der bewertenden Lesart (vgl. Beispiel 8) zeigen, nicht ausreichend.

Die Identifikation der unterspezifizierten Bedeutung ergibt sich also sowohl aus sequenziellen, die Teilnehmerperspektive berücksichtigenden Analysen als auch aus nur introspektiv bzw. anhand linguistischer Kenntnisse möglichen Uberlegungen. Da die Bedeutungsbestandteile von kommen (und gehen) in der Literatur auch ohne Bezug zur Frame-Semantik beschrieben werden, stellt sich die Frage, welchen Mehrwert eine framesemantische Beschreibung der von kommen stammenden Bedeutungsanteile ergibt. M. E. ist dieser vor allem deskriptiver Art: Ein Bezug auf das Frame-Element Goal macht eine konsistente Beschreibung der Partikel- und Verbvorkommen von $\operatorname{komm}(e n)$ möglich, die bei anders paraphrasierenden Bedeutungsbeschreibungen nicht notwendigerweise gegeben wäre. Es ergeben sich aber folgende Probleme: 1) Die Annahme einer Evokation des Motion-Frames auch durch die Partikel, wie sie eine kognitive Sicht nahelegt, erscheint unplausibel, da die metaphorische, abstrakte Konzeptualisierung des Ziels und der Wegfall der Bewegungsbedeutung lexikalisiert sind und nicht bei jeder Verwendung kontextuell konstruiert werden müssen. 2) Auch die Annahme eines anderen Frames ist schwierig: Wie sollte dieser definiert sein? Sollte er ein Frame-Element ,abstract Goal' oder,(kontextuell relevante) Handlung ' enthalten, das aber grundsätzlich nicht formal realisierbar ist? 3) Auch wenn man annimmt, dass eine Partikel einen Frame evozieren kann, ist die Annahme eines propositionalen Frames hier nicht plausibel, es ist nur ein funktionaler, handlungsbezogener Frame denkbar, wie ihn auch schon die Paraphrase der angenommenen unterspezifizierten Bedeutung formuliert (etwa: ,Aufforderung an ein Agens, eine Handlung umzusetzen', wobei Agens und Handlung Frame-Elemente wären); so etwas sieht die Frame-Semantik bisher aber nicht vor. Ob diese von deskriptivem oder explanativem Nutzen bei der Analyse von mündlichen Daten ist, hängt also nicht nur von der Methode (Sequenzanalyse vs. (korpusbasierte) Introspektion) ab, sondern auch vom Untersuchungsgegenstand (Verben vs. Partikeln). 


\section{Fazit}

Es ist aus interaktionsanalytischer Sicht unbestritten, dass common ground zur Bedeutungskonstitution beiträgt, und wenn man den Bezug zu diesem (bzw. dem nicht rein interaktionsgeschichtlichen Teil) als Frame-Evokation konzeptualisiert, geht man davon aus, dass dies eine kognitive Komponente hat, da sie ,im Kopf' der Sprecher und Rezipienten stattfindet und nicht immer ,angezeigt' wird. Aus semantiktheoretischer Sicht ist klar, dass die Zuordnung und Identifikation von Frames in der Analyse nie rein datengesteuert sein kann, da introspektive Erwägungen für eine systematische Erfassung unabdingbar sind und interaktionslinguistische Ansätze mit ihren Methoden keine vollständige Semantiktheorie erstellen können; die angesetzten Frames müssen ebenso wie ,klassische‘ Wörterbuchparaphrasen vom Analytiker und der Theorie (inkl. ihrem Erklärungszweck) vorgegeben werden, und dies ist auch in den wenigen vorhandenen interaktionsanalytischen Arbeiten zur Verbsemantik Praxis (vgl. z. B. Deppermann 2008b; Imo 2007). Eine dennoch so stark wie möglich datengestützte Systematisierung kann dafür sorgen, dass ein geeignetes Granularitätsniveau gefunden wird, d.h. die Möglichkeit der Trennung zwischen Lesarten empirisch validiert wird (vgl. dazu Zeschel 2017). Der Vorteil der Frame-Semantik ist es, eine kohärente, kognitiv plausible Theorie bereitzustellen, die zudem zu interaktionslinguistischen Ansätzen passt, weil beiden der Einbezug von Pragmatik und Wissensbeständen ein theoretisches Anliegen ist. Die kognitive Plausiblität der Frames und ihrer Zusammenhänge kann allerdings interaktionsanalytisch nicht belegt werden; dies erfordert psycholinguistische Methoden. Es zeigt sich zudem an den obigen Beispielen, dass die Frame-Semantik nicht ausreichend für die Erklärung aller Teile der Bedeutungskonstitution ist; viele Inferenzen beziehen sich auf über Frames hinausgehende, z. B. sequenzielle oder interaktionsgeschichtliche Aspekte des common ground. Deshalb ist generell im Falle interaktionaler Daten und insbesondere dann, wenn Partikeln der Untersuchungsgegenstand sind, eine Sequenzanalyse zwingend, eine Untersuchung der Bedeutungskonstitution also nicht ohne diese möglich. Außerdem lässt sich der bei Wandelphänomenen entstehende semantisch-pragmatische Mehrwert (wie im Fall der Partikeln) nicht automatisch aus Framehierarchien erklären, sondern muss in der einzelfallübergreifenden linguistischen Analyse gefunden werden, egal welches Semantikkonzept man voraussetzt. 


\section{Literatur}

Auer, Peter (2000): „Online-Syntax - Oder: was es bedeuten könnte, die Zeitlichkeit der mündlichen Sprache ernst zu nehmen “. In: Sprache und Literatur 31, 43-56.

Auer, Peter/Günthner, Susanne (2005): „Die Entstehung von Diskursmarkern im Deutschen - ein Fall von Grammatikalisierung?" In: Leuschner, Torsten/Mortelmans, Tanja (Hg.):

Grammatikalisierung im Deutschen. Berlin/New York: de Gruyter, 335-362.

Bücker, Jörg (2008): ,Elf Freunde sollt ihr sein? Von wegen!“ - nicht-präpositionale Spielarten mit von wegen als Projektorkonstruktionen in der deutschen Gegenwartssprache. Münster: GIDI Arbeitspapier 17. http://noam.uni-muenster.de/gidi/arbeitspapiere/arbeitspapier17. pdf [15.11.2017]

Busse, Dietrich (2012): Frame-Semantik. Ein Kompendium. Berlin/Boston: de Gruyter. Clark, Herbert H. (1996): Using Language. Cambridge: Cambridge University Press.

Deppermann, Arnulf (2002): „Von der Kognition zur verbalen Interaktion: Bedeutungskonstitution im Kontext aus Sicht der Kognitionswissenschaften und Gesprächsforschung “. In: Deppermann/Spranz-Fogasy (Hg.) (2002): be-deuten. Wie Bedeutung im Gespräch entsteht. Tübingen: Stauffenburg, 11-33.

Deppermann, Arnulf (2007): Grammatik und Semantik aus gesprächsanalytischer Sicht. Berlin/ New York: de Gruyter.

Deppermann, Arnulf (2008a): „Verstehen im Gespräch“. In: Kämper, Heidrun/Eichinger, Ludwig M. (Hg.): Sprache, Kognition, Kultur: Sprache zwischen mentaler Struktur und kultureller Prägung. Berlin/New York: de Gruyter, 225-261.

Deppermann, Arnulf (2008b): „Lexikalische Bedeutung oder Konstruktionsbedeutungen? Eine Untersuchung am Beispiel von Konstruktionen mit verstehen". In: Stefanowitsch, Anatol/ Fischer, Kerstin ( $\mathrm{Hg}$.): Konstruktionsgrammatik Bd. 2: Von der Konstruktion zur Grammatik. Tübingen: Stauffenburg, 103-133.

Deppermann, Arnulf (2012): „How does ,cognition“ matter to the analysis of talk-ininteraction?" In: Language Sciences 34(6), 746-767.

Deppermann, Arnulf/Helmer, Henrike (2013): „Zur Grammatik des Verstehens im Gespräch: Inferenzen anzeigen und Handlungskonsequenzen ziehen mit also und dann“. In: Zeitschrift für Sprachwissenschaft 32(1), 1-40.

Deppermann, Arnulf/Spranz-Fogasy (Hg.) (2002): be-deuten. Wie Bedeutung im Gespräch entsteht. Tübingen: Stauffenburg.

Di Meola, Claudio (1994): Kommen und gehen. Eine kognitiv-linguistische Untersuchung der Polysemie deiktischer Bewegungsverben. Tübingen: Niemeyer.

Fillmore, Charles J. (1982): „Frame Semantics“. In: Linguistics in the Morning Calm. The Linguistic Society of Korea: Seoul, 111-137.

Fillmore, Charles J. (1985): „Frames and the semantics of understanding“. In: Quaderni di Semantica 6, 222-254.

Fillmore, Charles J./Johnson, Christopher R./Petruck, Miriam R. L. (2003): „Background to FrameNet“. In: International Journal of Lexicography 16(3), 235-250.

Gohl, Christine/Günthner, Susanne (1999): „Grammatikalisierung von weil als Diskursmarker in der gesprochenen Sprache“. In: Zeitschrift für Sprachwissenschaft 18(1), 39-75.

Günthner, Susanne (1999): „Entwickelt sich der Konzessivkonnektor obwohl zum Diskursmarker? Grammatikalisierungstendenzen im gesprochenen Deutsch“. In: Linguistische Berichte 180, 409-446. 
Heritage, John (1984): „A change-of-state token and aspects of its sequential placement“. In: Atkinson, J. Maxwell/Heritage, John (Hg.): Structures of Social Action. Cambridge: Cambridge University Press, 299-345.

Imo, Wolfgang (2007): Construction Grammar und Gesprochene-Sprache-Forschung. Konstruktionen mit zehn matrixsatzfähigen Verben im gesprochenen Deutsch. Tübingen: Niemeyer.

Imo, Wolfgang (2014): „Interaktionale Linguistik“. In: Staffeldt, Seven/Hagemann, Jörg (Hg.): Pragmatiktheorien. Analysen im Vergleich. Tübingen: Stauffenburg, 49-82.

Nübling, Damaris (2004): „Die prototypische Interjektion: Ein Definitionsvorschlag“. In: Zeitschrift für Semiotik 26, 11-45.

Potter, Jonathan/Edwards, Derek (2013): „Conversation Analysis and Psychology“. In: Sidnell, Jack/Stivers, Tanya (Hg.): The Handbook of Conversation Analysis. Malden, Mass.: WileyBlackwell, 701-725.

Proske, Nadine (2014): „ $\mathrm{h}$ ach KOMM; hör AUF mit dem klEInkram. - die Partikel komm zwischen Interjektion und Diskursmarker". In: Gesprächsforschung - Online-Zeitschrift zur verbalen Interaktion 15, 121-160.

Proske, Nadine (2016): „Zur Perspektivierung von verbalen Handlungen und kognitiven Prozessen durch die Verwendung von Bewegungsverben im gesprochenen Deutsch". In: Kreuz, Christian/Mroczynski, Robert (Hg.): Sprache, Kultur, Mentalität. Sprach- und kulturwissenschaftliche Beiträge zur Analyse von Mentalitäten. Berlin: LIT, 231-273.

Proske, Nadine (2017a): „Perspektivierung von Handlungen und Zuschreibung von Intentionalität durch pseudokoordiniertes kommen". In: Deppermann, Arnulf/Proske, Nadine/ Zeschel, Arne (Hg.): Verben im interaktiven Kontext. Bewegungsverben und mentale Verben im gesprochenen Deutsch. Tübingen: Narr, 177-247.

Proske, Nadine (2017b): „Ankündigungen und Thematisierungen von Themenwechseln mit kommen und gehen in institutionellen und öffentlichen Interaktionen “. In: Deppermann, Arnulf/Proske, Nadine/Zeschel, Arne (Hg.): Verben im interaktiven Kontext. Bewegungsverben und mentale Verben im gesprochenen Deutsch. Tübingen: Narr, 117-175.

Reisigl, Martin (1999): Sekundäre Interjektionen. Eine diskursanalytische Annäherung. Frankfurt am Main: Lang.

Ruppenhofer, Josef et al. (2010): „FrameNet II. Extended Theory and Practice“. https:// framenet2.icsi.berkeley.edu/docs/r1.5/book.pdf [15.11.2017]

Sacks, Harvey/Schegloff, Emanuel A./Jefferson, Gail (1974): „A simplest systematics for the organisation of turn taking in conversation". In: Language 50(4), 696-735.

Schumacher, Helmut et al. (2004): VALBU - Valenzwörterbuch deutscher Verben. Tübingen: Narr.

Selting, Margret et al. (2009): „Gesprächsanalytisches Transkriptionssystem 2 (GAT 2)“. In: Gesprächsforschung - Online-Zeitschrift zur verbalen Interaktion 10, 353-402.

Sullivan, Karen (2013): Frames and Constructions in Metaphoric Language. Amsterdam/ Philadelphia: Benjamins.

Tomasello, Michael (2008): Origins of Human Communication. Cambridge: Cambridge University Press.

Wahrig-Burfeind, Renate ('2012): Deutsches Wörterbuch. Gütersloh/München: Bertelsmann. Zeschel, Arne (2017): „Kommen und gehen im gesprochenen Deutsch“. In: Deppermann, Arnulf/Proske, Nadine/Zeschel, Arne ( $\mathrm{Hg}$.$) : Verben im interaktiven Kontext.$ Bewegungsverben und mentale Verben im gesprochenen Deutsch. Tübingen: Narr, 41-116. 
Zeschel, Arne/Proske, Nadine (2015): „Usage-based linguistics and conversational interaction. A case study of German motion verbs“. In: Yearbook of the German Cognitive Linguistics Association, 123-144.

Ziem, Alexander (2015): „Nullinstanziierungen im gesprochenen Deutsch: Kohärenz durch grammatische Konstruktionen?"“ In: Bücker, Jörg/Günthner, Susanne/Imo, Wolfgang ( $\mathrm{Hg}$.): Konstruktionsgrammatik V. Konstruktionen im Spannungsfeld von sequenziellen Mustern, kommunikativen Gattungen und Textsorten. Tübingen: Stauffenburg, 45-80. 